\title{
Brain Glucose Metabolism, Cognition, and Cardiorespiratory Fitness Following Exercise Training in Adults at Risk for Alzheimer's Disease
}

\author{
Julian M. Gaitán ${ }^{\mathrm{a}}$, Elizabeth A. Boots ${ }^{\mathrm{a}, 1}$, Ryan J. Dougherty ${ }^{\mathrm{a}, \mathrm{b}, \mathrm{c}}$, Jennifer M. Oh $^{\mathrm{a}, \mathrm{d}}$, Yue Ma ${ }^{\mathrm{a}}$, \\ Dorothy F. Edwards ${ }^{\mathrm{a}, \mathrm{d}, \mathrm{e}}$, Bradley T. Christian ${ }^{\mathrm{a}, \mathrm{f}}$, Dane B. Cook ${ }^{\mathrm{b}, \mathrm{c}}$ and Ozioma C. Okonkwo ${ }^{\mathrm{a}, \mathrm{d}, \mathrm{e}, *}$ \\ ${ }^{a}$ Wisconsin Alzheimer's Disease Research Center, University of Wisconsin School of Medicine \\ and Public Health, Madison, WI, USA \\ ${ }^{\mathrm{b}}$ Department of Kinesiology, University of Wisconsin School of Education, Madison, WI, USA \\ ${ }^{\mathrm{c}}$ William S. Middleton Memorial Veterans Hospital, Madison, WI, USA \\ ${ }^{\mathrm{d}}$ Geriatric Research Education and Clinical Center, William S. Middleton Memorial Veterans Hospital, \\ Madison, WI, USA \\ ${ }^{\mathrm{e}}$ Wisconsin Alzheimer's Institute, University of Wisconsin School of Medicine and Public Health, \\ Madison, WI, USA \\ ${ }^{\mathrm{f}}$ Department of Medical Physics, University of Wisconsin School of Medicine and Public Health, \\ Madison, WI, USA
}

\begin{abstract}
Aerobic exercise has been associated with reduced burden of brain and cognitive changes related to Alzheimer's disease (AD). However, it is unknown whether exercise training in asymptomatic individuals harboring risk for AD improves outcomes associated with AD. We investigated the effect of 26 weeks of supervised aerobic treadmill exercise training on brain glucose metabolism and cognition among 23 late-middle-aged adults from a cohort enriched with familial and genetic risk of AD. They were randomized to Usual Physical Activity (PA) or Enhanced PA conditions. Usual PA received instruction about maintaining an active lifestyle. Enhanced PA completed a progressive exercise training program consisting of 3 sessions of treadmill walking per week for 26 weeks. By week seven, participants exercised at $70-80 \%$ heart rate reserve for 50 minutes per session to achieve 150 minutes of moderate intensity activity per week in accordance with public health guidelines. Before and after the intervention, participants completed a graded treadmill test to assess $\mathrm{VO}_{2}$ peak as a measure of cardiorespiratory fitness (CRF), wore an accelerometer to measure free-living PA, underwent ${ }^{18} \mathrm{~F}$-fluorodeoxyglucose positron emission tomography imaging to assess brain glucose metabolism, and a neuropsychological battery to assess episodic memory and executive function. $\mathrm{VO}_{2}$ peak increased, sedentary behavior decreased, and moderate-to-vigorous PA increased significantly in the Enhanced PA group as compared to Usual PA. Glucose metabolism in the posterior cingulate cortex (PCC) did not change significantly in Enhanced PA relative to Usual PA. However, change in PCC glucose metabolism correlated positively with change in $\mathrm{VO}_{2}$ peak. Executive function, but not episodic memory, was significantly improved after
\end{abstract}

\footnotetext{
*Correspondence to: Ozioma Okonkwo, PhD, Department of Medicine and Alzheimer's Disease Research Center, University of Wisconsin School of Medicine and Public Health, 600 Highland Ave. J5/1M CSC MC2420, Madison, WI 53792, USA. Tel.: +1608 265 4479; Fax: +1 608265 3091; E-mail: ozioma@ medicine.wisc.edu.
}

\footnotetext{
${ }^{1}$ Present address: Department of Psychology, University of Illinois at Chicago, Chicago, IL, USA, and Rush Alzheimer's Disease Research Center, Rush University Medical Center, Chicago, IL, USA.
} 
Enhanced PA relative to Usual PA. Improvement in executive function correlated with increased $\mathrm{VO}_{2}$ peak. Favorable CRF adaptation after 26 weeks of aerobic exercise training was associated with improvements in PCC glucose metabolism and executive function, important markers of AD.

Keywords: Aerobic exercise, physical activity, Alzheimer's disease, posterior cingulate cortex, executive function, episodic memory

\section{INTRODUCTION}

The human and monetary costs of Alzheimer's disease $(\mathrm{AD})$ are concerning given that the prevalence of $\mathrm{AD}$ is expected to increase dramatically in the coming years [1]. While disease-modifying agents are under investigation, attention is shifting toward prevention of $\mathrm{AD}$ especially via non-pharmacological approaches [2]. Furthermore, the preclinical phase is now believed to be the most opportune window during which to modify the disease course prior to substantial neuronal damage [2].

Aerobic exercise training is a low-cost, low-risk intervention that may delay disease progression. In animal models, exercise has been shown to alter the pathophysiology of AD [3-5]. Furthermore, mounting evidence from randomized controlled trials reveals that aerobic exercise training in older adults can affect outcomes associated with $\mathrm{AD}$ including brain volume, memory, and executive function [6-8] although effect sizes may be small to medium [9]. The mechanisms by which exercise benefits brain health (e.g. brain volume) and supports cognition have yet to be fully elucidated. Cross-sectional, animal model, and limited human intervention data support that cardiorespiratory fitness (CRF) is related to possible mechanisms that support brain health such as angiogenesis $[10,11]$ and white matter/brain structure integrity [12-14]. Nonetheless, whether aerobic exercise training affects key biomarkers of $\mathrm{AD}$ remains unknown. While asymptomatic individuals with genetic and familial history of AD face increased risk of developing $\mathrm{AD}[15,16]$, it also remains unknown whether aerobic exercise training modulates $\mathrm{CRF}$ and outcomes associated with $\mathrm{AD}$ among such individuals. This is important because interventions to alter $\mathrm{AD}$ pathophysiology are more likely to be effective if implemented prior to significant neuronal damage [2] and because targeting at-risk individuals lends itself to reducing the growing population with AD.

Posterior cingulate cortex (PCC) hypometabolism is an established characteristic of $\mathrm{AD}$ that appears early in the disease course and is suggestive of neuronal damage [17]. PCC hypometabolism can even be detected in cognitively normal individuals with increased risk for the disease [18]. While evidence supports that aerobic exercise benefits brain structure and function, how brain glucose metabolism responds to aerobic exercise training among individuals at risk for AD remains unknown. Furthermore, episodic memory and executive function are cognitive domains relevant to $\operatorname{AD}[19,20]$ that can be improved with aerobic exercise [6, 8]. Disturbance in executive function has been shown to be present in cognitively normal individuals with AD pathology, sometimes prior to detectable memory deficit. The color-word interference (i.e., Stroop) effect may be a particularly relevant measure of executive function for identifying pre-clinical AD [20].

In this study, we investigated whether engagement in 26 weeks of aerobic exercise training favorably alters brain glucose metabolism and cognition in asymptomatic, late-middle-aged adults at risk for $\mathrm{AD}$. The hypothesis was that participants performing aerobic exercise training would preserve brain glucose metabolism, memory, and executive function while scores in the comparison group would decline.

\section{METHODS}

\section{Participants}

Twenty-three asymptomatic, late-middle-aged adults were included in this pilot randomized, controlled, 26-week aerobic exercise training intervention. Participants were recruited from the Wisconsin Registry for Alzheimer's Prevention (WRAP), a cohort enriched for family history of $\mathrm{AD}$ and the APOE $\varepsilon 4$ allele [21], or the Wisconsin Alzheimer's Disease Research Center (WADRC) Investigating Memory in People At risk, Causes and Treatments (IMPACT) cohort. Thirty-eight (WRAP) to $40 \%$ (WADRC IMPACT) of participants in these cohorts carry at least one APOE $\varepsilon 4$ allele as compared to approximately $15 \%$ in a population 


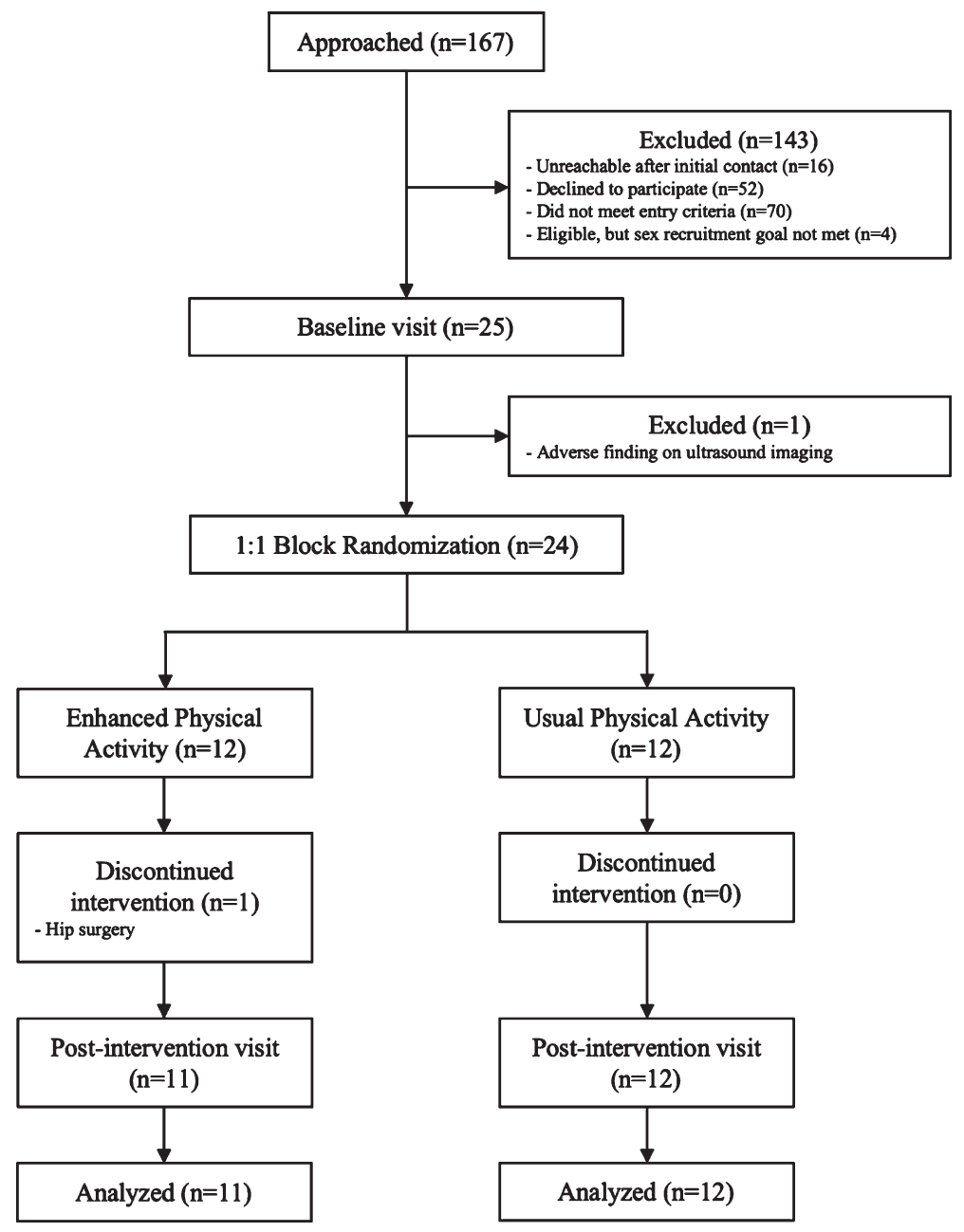

Fig. 1. Recruitment and study protocol flowchart.

of comparable age [22]. To be eligible for our study, participants were required to be between ages 45 to 80 , have a documented positive parental history of $\mathrm{AD}$, be deemed cognitively healthy by a consensus panel consisting of at least of a physician, a neuropsychologist, and one other clinician (e.g., a nurse practitioner), and be physically inactive $(<150 \mathrm{~min} /$ week moderate intensity activity) according to a telephone assessment of physical activity (PA) [23]. Exclusion criteria were pregnancy, diabetes, presence of significant neurological disease, known structural brain abnormalities, contraindication to magnetic resonance imaging (MRI), medical condition prohibiting safe participation in aerobic exercise training, severe psychiatric conditions or history of substance abuse, any significant medical condition affecting cognition, severe untreated hypertension, use of antipsychotic medications, and cancer in the previous five years. Figure 1 details the enrollment process for the study. All study procedures were completed at baseline and post-intervention, as described below. Participants gave written informed consent prior to study entry and the study was approved by the University of Wisconsin - Madison Institutional Review Board. The study was registered with ClinicalTrials.gov, number NCT02384993.

\section{Randomization and intervention}

Participants were block randomized using a 1:1 scheme, stratified for age and sex, into either Usual PA or Enhanced PA. Participants randomized to Usual PA received informal education at the time of 
randomization about the importance of maintaining a healthy and active lifestyle. They were then provided with a copy of "Exercise \& Physical Activity: Your Everyday Guide from the National Institute on Aging." No additional guidance or instruction about an exercise program was given to this group during the study.

Participants in the Enhanced PA group completed three sessions per week of supervised treadmill walking for 26 weeks. Session duration increased by five minutes each week from 15 minutes in week one to 40 minutes in week six, and finally 50 minutes in weeks seven to 26 . Treadmill speed and incline was adjusted by the supervising exercise specialist to achieve 50-60\% heart rate reserve (HRR) for weeks one and two, 60-70\% HRR for weeks three through six, and 70-80\% HRR for weeks seven through 26. The intent of this structured program was to incrementally increase exercise duration and intensity, given that these individuals had previously engaged in little PA. Thus, beginning in week seven and continuing through week 26, participants completed 150 minutes per week of moderate intensity aerobic exercise in accordance with public health PA guidelines [24]. If a participant could not attend an exercise session(s) due to travel, they were asked to complete any missed sessions independently or to reschedule sessions with the exercise specialist. Heart rate (HR) and rating of perceived exertion (RPE; [25]) were assessed by the exercise specialist every five minutes throughout each session. Exercise sessions began and ended with five-minute warm up and cool down periods.

\section{Clinical assessment}

Participants reported to the University of Wisconsin - Madison Clinical Research Unit in the morning after a 12-hour fast from food, nicotine, caffeine, alcohol, and medications with vasomodulatory properties. Blood pressure was obtained three times after the participant was seated for 10 minutes and the average of the second and third readings was recorded for systolic and diastolic blood pressure. Weight was measured to the nearest tenth of a kilogram using a medical grade scale and height was measured using a stadiometer. All participants already had parental history of AD and APOE genetic status on record [21]. Fasting blood samples were collected from the antecubital vein and sent to the UW Health Central Laboratory for analysis of clinical laboratory values.

\section{Cardiorespiratory fitness}

Participants performed a continuous graded peak oxygen consumption $\left(\mathrm{VO}_{2}\right.$ peak) test on a treadmill (Trackmaster TMX428CP, Full Vision Inc., Newton, KS) to assess CRF after a 12-hour fast from nicotine, caffeine, alcohol, and medication (prescription and over-the-counter). Participants refrained from exercise on the day of the test. The graded treadmill test was administered by a certified exercise physiologist and supervised by a physician. During a brief warm-up period, a comfortable walking speed was determined that was kept constant for the duration of testing. The majority of participants walked at 3.5 miles per hour; however, if the participant indicated that speed to be uncomfortable, a slower speed was chosen. The test began with an incline of $0 \%$, and the incline was increased by $2.5 \%$ every two minutes until the participant reached volitional exhaustion or the supervising exercise physiologist stopped the test due to safety concerns.

A metabolic cart and 2-way non-rebreathing valve (TrueOne 2400, ParvoMedics, Sandy, UT) were used to obtain continuous measurements of $\mathrm{VO}_{2}$, $\mathrm{VCO}_{2}$, and minute ventilation. The system was calibrated prior to each test using standard gases with known concentrations and with a calibrated threeliter syringe. HR via electrocardiogram and RPE were recorded during the last 30 seconds of each stage. Criteria for meeting $\mathrm{VO}_{2}$ peak involved satisfying at least two of the following: respiratory exchange ratio $\geq 1.1$, achievement of $90 \%$ of agepredicted maximum $\mathrm{HR}, \mathrm{RPE} \geq 17$, and a change in $\mathrm{VO}_{2}<200 \mathrm{~mL}$ with an increase in treadmill incline [26]. Following volitional exhaustion, the speed and grade of the treadmill were reduced to $2 \mathrm{mph}$ and $0 \%$ grade for a 3-minute active recovery period.

\section{Accelerometry}

Participants wore a triaxial accelerometer (GT3X+, Actigraph LLC, Pensacola, FL) for seven consecutive days to record free-living $\mathrm{PA}$ and sedentary behavior before and after the intervention. The device was given to participants at the end of the post-intervention visit such that accelerometer data collection was done the week following the completion of the exercise intervention and all other post-intervention testing. The accelerometer was worn on the hip during all waking hours except while bathing or swimming. Participants kept a written $\log$ detailing when they wore the device 
and when they exercised or did any other activity. This facilitated the interpretation of accelerometer data by the study team. Standard accelerometer data inclusion criteria were used: at least 10 hours of valid wear time per day for a minimum of three weekdays and one weekend day [27]. Accelerometer data in 1-second epochs were processed using the sojourn-3 axis method [28] to calculate time spent sedentary and in light (LPA), moderate (MPA), and vigorous (VPA) intensity PA [28, 29]. Briefly, this method utilizes data from vertical, anterior-posterior, and medial-lateral axes to identify independent bout intervals - time between starting one activity and transitioning to another-by instances of rapid acceleration or deceleration.

Within the sojourn-3 axis method [28], estimated metabolic equivalents (METs) are determined for each bout interval using a validated neural network approach [30], as opposed to the traditional counts-per-minute approach. The METs values were classified into PA intensity categories: $<1.5$ METs = sedentary, 1.5-2.99 METs = LPA, 3-6 METs $=$ MPA, and $>6$ METs $=$ VPA. Moderate and vigorous PA were collapsed into a composite score of moderate-to-vigorous PA (MVPA) as our intent was to determine whether participants were engaged in PA aligned with public health guidelines that recommend moderate and/or vigorous PA [24]

\section{Neuroimaging protocol}

Participants underwent ${ }^{18}$ F-fluorodeoxyglucose (FDG) positron emission tomography (FDG-PET) imaging after a four-hour fast from food, nicotine, caffeine, and alcohol. Details of the FDG-PET scan have been published previously [31-33]. Images were acquired on a Siemens EXACT HR+ scanner (Siemens AG, Erlangen, Germany) using the Alzheimer's Disease Neuroimaging Initiative (ADNI) protocol [34] which involves a $5 \mathrm{mCi}$ FDG injection. Imaging began after a 30-minute uptake period, and the scan was a 30-minute transaxial acquisition. Post-processing was done using an automated pipeline [35]. Each FDG-PET image was proportionally scaled to the mean FDG signal from the cerebellar vermis and pons. We focused on the posterior cingulate cortex (PCC), a well-established inception site for AD-related neurometabolic alterations [36], and sampled FDG uptake values from it using the PCC mask within the ADNI FDG Meta-ROI suite [37]. The mean FDG uptake in the entire PCC was calculated for each group for statistical analysis.

\section{Cognitive function}

A trained technician administered the cognitive test battery to each participant. These tests targeted global cognition, as well as cognitive domains of episodic verbal memory and executive function. The test battery included the Mini Mental State Examination (MMSE) [38], California Verbal Learning Test-II (CVLT) [39], and the Delis-Kaplan Executive Function System (D-KEFS) which comprises Verbal Fluency, Design Fluency, Trail Making, Sorting, Color-Word Interference (CWI), and Tower subtests [40]. For the D-KEFS, only the CWI subtest was analyzed for the present report due to its particular sensitivity to executive dysfunction in pre-clinical AD [20].

\section{Statistical analyses}

Data were analyzed using SPSS 25.0 (IBM Corp., Armonk, NY). Independent samples $t$-tests (for continuous variables) and Fischer's exact tests (for categorical variables) were used to analyze baseline differences between treatment groups. For each outcome, we calculated change from baseline to postintervention. Then, independent samples $t$-tests was used to analyze differences between groups on the change measure. The change in PCC FDG uptake was not normally distributed within the Usual PA group. The data for this variable were thus transformed using Blom's formula prior to being analyzed using the $t$-test. Pearson's correlation was used to determine associations between outcomes. Data are presented as mean (standard deviation), and significance was established as $p<0.05$.

\section{RESULTS}

\section{Baseline characteristics}

The Usual and Enhanced PA groups were composed of late-middle aged participants with an overweight BMI on average. Participants had normal cognition as demonstrated by their MMSE scores and had on average 16 years of schooling. Usual and Enhanced PA groups were similar in age, sex, years of schooling, APOE positivity, parental history of AD, cognitive capacity, body habitus, and blood pressure. Table 1 presents the characteristics of the participants within Usual and Enhanced PA at baseline. Furthermore, Table 1 shows that the randomization procedure successfully produced groups that did not 
Table 1

Baseline characteristics of the participants

\begin{tabular}{lccc}
\hline Variable & Usual PA, $n=12$ & Enhanced PA, $n=11$ & $p$ \\
\hline Characteristics & & & \\
Age, y & $63.92(5.19)$ & $65.88(4.00)$ & 0.33 \\
Female, $\mathrm{n}$ & 6 & 5 & 0.99 \\
Education, y & $16.67(2.57)$ & $16.27(2.33)$ & 0.71 \\
Parental history of AD, $\mathrm{n}$ & 12 & 11 & - \\
APOE $\varepsilon 4$ positive, $\mathrm{n}$ & 5 & 3 & 0.67 \\
MMSE, a.u. & $29.67(0.49)$ & $29.55(0.69)$ & 0.63 \\
BMI, kg/m & $29.38(5.96)$ & $29.89(4.81)$ & 0.82 \\
Systolic BP, mmHg & $128.50(19.65)$ & $123.23(18.65)$ & 0.52 \\
Diastolic BP, mmHg & $79.92(9.47)$ & $75.96(10.45)$ & 0.35 \\
Outcomes & & & \\
VO ${ }_{2}$ peak, mL/kg/min & $25.74(6.58)$ & $23.57(4.97)$ & 0.39 \\
Sedentary behavior, min & $670.73(94.26)$ & $725.96(75.81)$ & 0.14 \\
MVPA, min & $78.97(35.29)$ & $60.58(23.49)$ & 0.16 \\
Brain glucose metabolism, a.u. & $1.49(0.12)$ & $1.50(0.11)$ & 0.84 \\
CVLT Total, words & $49.58(11.06)$ & $53.18(7.99)$ & 0.39 \\
CVLT Long Delay, words & $10.83(2.82)$ & $12.00(2.72)$ & 0.33 \\
D-KEFS CWI, seconds & $60.00(11.71)$ & $64.82(15.11)$ & 0.40 \\
\hline
\end{tabular}

Characteristics of the participants at study entry. $p$-values result from $t$-tests and Fischer's exact tests to determine differences between groups. Data are presented as mean (standard deviation). Abbreviations: physical activity (PA); Alzheimer's disease (AD); apolipoprotein E epsilon 4 (APOE $\varepsilon 4$ ); Mini-Mental State Examination (MMSE); body mass index (BMI); blood pressure (BP); peak oxygen consumption $\left(\mathrm{VO}_{2}\right.$ peak).

differ at baseline on the outcome measures, including $\mathrm{VO}_{2}$ peak, sedentary behavior and MVPA, brain glucose metabolism, and cognitive performance.

\section{Exercise training characteristics}

Participants in the Enhanced PA group successfully engaged in moderate intensity aerobic exercise with mean HRR and RPE over 73\% and 12 a.u., respectively, during their 50-minute exercise sessions. Table 2 presents summary data for exercise intensity, volume, and adherence for participants in the Enhanced PA group. Data in Table 2 represent group means from weeks 7-26 of the intervention because weeks 1-6 consisted of a gradual increase of session duration and intensity. Six of the 11 participants randomized to Enhanced PA missed at least one supervised exercise session due to travel or illness. Participants missed an average of 2.4 and a maximum of 10 (by a single participant) supervised sessions over the course of the program, and the maximum number of supervised sessions missed by a single participant was 10 . In all, participants missed 26 sessions during the intervention. Of these, they made up 19 sessions (e.g., by independently exercising while traveling), resulting in only 7 uncompleted sessions. Thus, 851 out of 858 total expected sessions were completed, resulting in a $99.2 \%$ session adherence rate.
Table 2

Enhanced PA aerobic exercise intervention characteristics

\begin{tabular}{lc}
\hline Variable & Enhanced PA, $n=11$ \\
\hline HR, bpm & $128.8(2.6)$ \\
HRR, \% & $73.5(2.5)$ \\
Treadmill speed, mph & $3.3(0.3)$ \\
Treadmill incline, \% & $7.7(3.3)$ \\
Session RPE, a.u. & $12.1(1.5)$ \\
Session adherence, \% & 99.2 \\
\hline
\end{tabular}

Descriptive statistics of the Enhanced PA intervention. Data are from weeks 7-26 (full exercise dose) of the intervention, as weeks 1-6 were comprised of a gradual buildup of session duration and intensity. Session adherence \% is presented for the full 26 weeks of the intervention. Data are presented as mean (standard deviation). Abbreviations: physical activity (PA); heart rate (HR); heart rate reserve (HRR); rating of perceived exertion (RPE).

\section{Accelerometry}

Sedentary behavior decreased from baseline to post- intervention from $726.0 \pm 22.9 \mathrm{~min} /$ day to $698.6 \pm 27.1 \mathrm{~min} /$ day in the Enhanced PA group and increased from $670.7 \pm 27.2 \mathrm{~min} /$ day to $690.1 \pm 26.7 \mathrm{~min} /$ day in the Usual PA group. The decline in sedentary behavior among Enhanced PA was significant as compared to the change in the Usual PA group, $p=0.003$ (Fig. 3A). Moderate-tovigorous PA (MVPA) increased in the Enhanced PA group from $60.58 \pm 7.08 \mathrm{~min} /$ day to $89.05 \pm$ $15.48 \mathrm{~min} /$ day and decreased in the Usual PA group from $78.97 \pm 10.19 \mathrm{~min} /$ day to $69.3 \pm 8.37 \mathrm{~min} /$ day. 


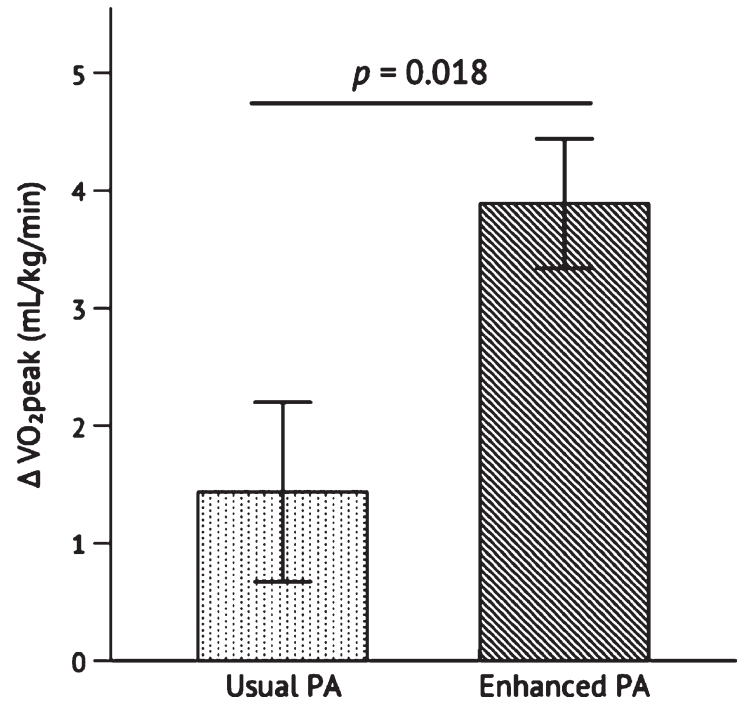

Fig. 2. Change in $\mathrm{VO}_{2}$ peak after the 26-week intervention. Independent samples $t$-test was conducted to determine the statistical significance of the difference between Usual PA $(n=12)$ and Enhanced PA $(n=11)$. Abbreviations: peak oxygen consumption ( $\mathrm{VO}_{2}$ peak); physical activity (PA).

The increase in MVPA in the Enhanced PA group was significant as compared to the change in the Usual PA group, $p=0.008$ (Fig. 3B).

\section{Cardiorespiratory fitness}

$\mathrm{VO}_{2}$ peak increased from baseline to postintervention from $23.57 \pm 4.97 \mathrm{~mL} / \mathrm{kg} / \mathrm{min}$ to $27.46 \pm 4.71 \mathrm{~mL} / \mathrm{kg} / \mathrm{min}$ in the Enhanced PA group, and from $25.74 \pm 6.58 \mathrm{~mL} / \mathrm{kg} / \mathrm{min}$ to $27.18 \pm 6.05 \mathrm{~mL} / \mathrm{kg} / \mathrm{min}$ in the Usual PA group. The increase in $\mathrm{VO}_{2}$ peak was significantly greater in Enhanced PA than Usual PA, $p=0.018$ (Fig. 2). The change in MVPA from baseline to post-intervention was not significantly correlated with change in $\mathrm{VO}_{2}$ peak, $r=0.129, p=0.557$.

\section{Cerebral glucose metabolism}

Because only change in PCC FDG (but not preor post-intervention values) was Blom-transformed due to non-normality (see METHODS: STATISTICAL ANALYSIS), raw values are presented as follows: PCC FDG uptake changed from baseline to post- intervention in the Enhanced PA group from $1.50 \pm 0.03$ a.u. to $1.54 \pm 0.04$ a.u. and changed in the Usual PA group from $1.49 \pm 0.04$ a.u. to $1.52 \pm 0.04$ a.u. Blom-transformed change in PCC FDG uptake was not different between groups,
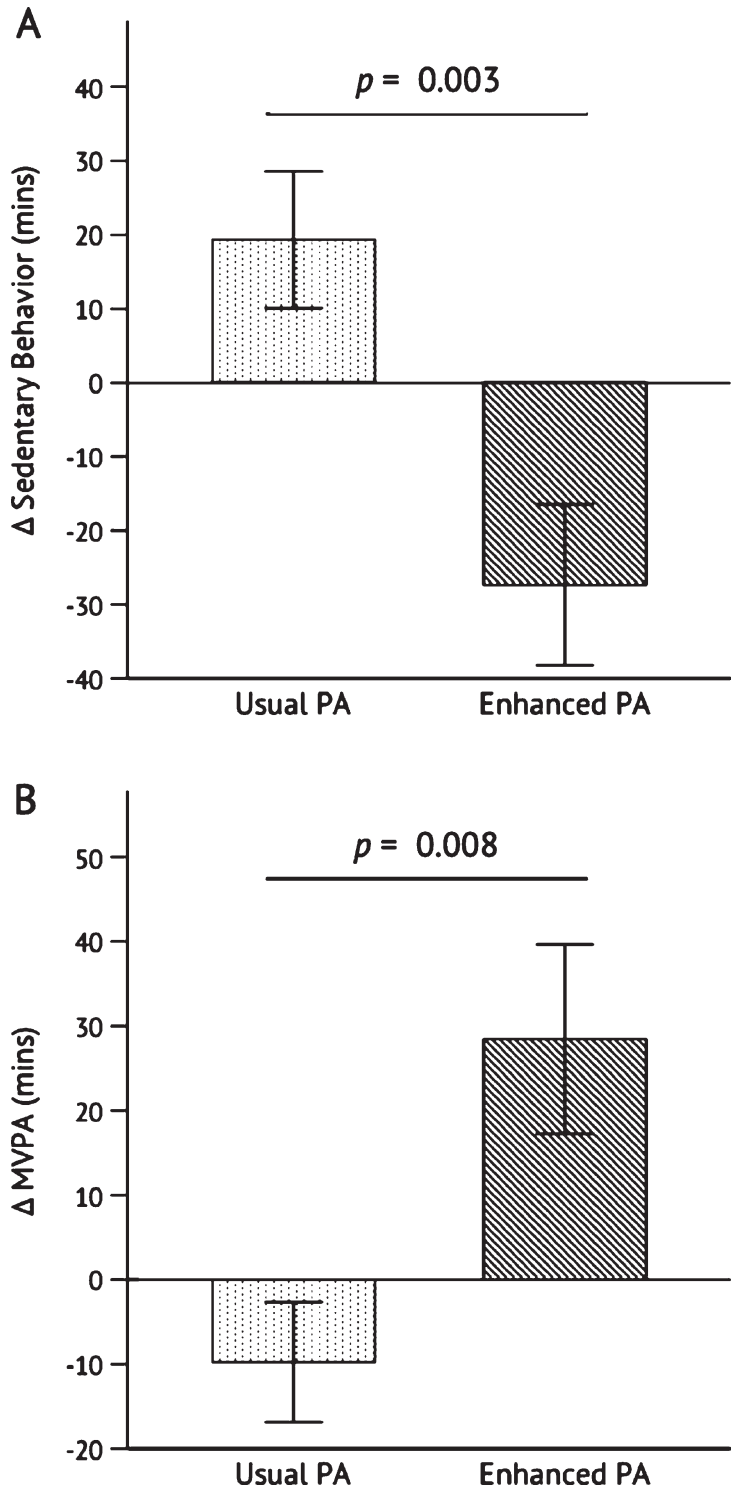

Fig. 3. Change in sedentary behavior (A) and moderate-tovigorous physical activity (B) after the 26-week intervention. Independent samples $t$-test was conducted to determine the statistical significance of the difference between Usual PA $(n=12)$ and Enhanced PA $(n=11)$. Abbreviations: moderate-to-vigorous physical activity (MVPA); physical activity (PA).

$p=0.482$ (Fig. 4). However, Pearson's correlation with data from both groups pooled revealed that Blom-transformed change in PCC FDG uptake was positively correlated, albeit not significantly, with change in $\mathrm{VO}_{2}$ peak, $r=0.381, p=0.073$ (Fig. 5); it was not significantly correlated with change from baseline to post-intervention MVPA, $r=0.200$, $p=0.360$. 


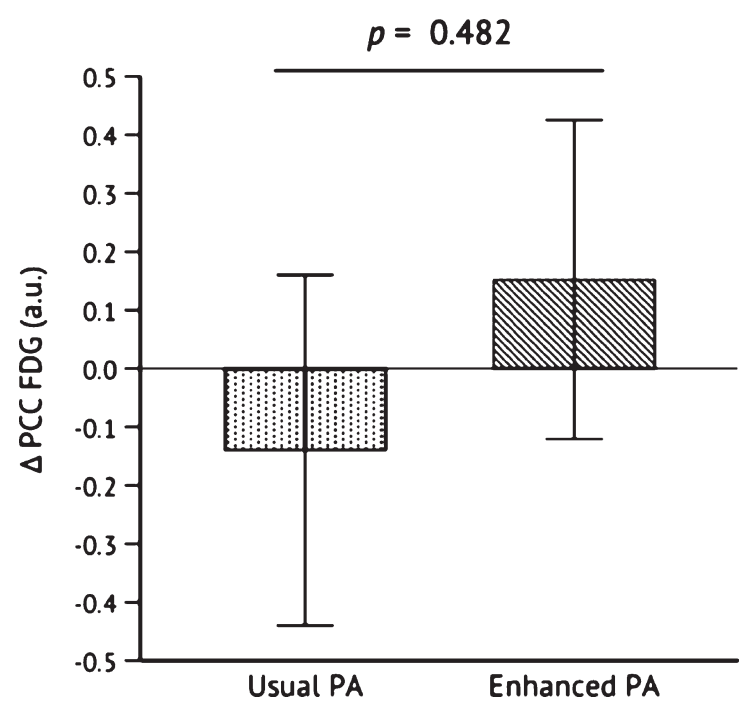

Fig. 4. Change in PCC FDG uptake after the 26-week intervention. Independent samples $t$-test was conducted to determine the statistical significance of the difference between Usual PA $(n=12)$ and Enhanced PA $(n=11)$. Abbreviations: Posterior cingulate cortex ${ }^{18}$ F-fluorodeoxyglucose (PCC FDG); physical activity (PA).

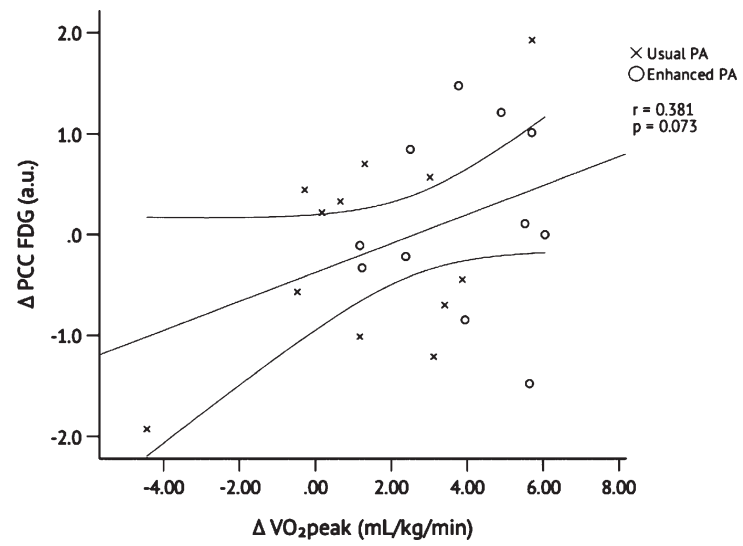

Fig. 5. Pearson's correlation between changes in PCC FDG uptake and $\mathrm{VO}_{2}$ peak after 26 weeks of Usual PA $(n=12)$ or Enhanced PA $(n=11)$. Abbreviations: Posterior cingulate cortex ${ }^{18}$ F-fluorodeoxyglucose (PCC FDG); peak oxygen consumption ( $\mathrm{VO}_{2}$ peak); physical activity (PA).

\section{Cognitive function}

Scores on the baseline MMSE indicated normal cognition in Enhanced and Usual PA and are presented in Table 1. Table 3 presents results of cognitive testing within each group, as well as effect sizes within and between groups. Higher scores on the CVLT indicate more words recalled and thus better performance. In contrast, lower scores on the D-KEFS CWI indicate faster responses and thus better performance. CVLT Total and Long Delay scores increased from baseline to post-intervention in both groups. D-KEFS CWI scores decreased from baseline to post- intervention in the Enhanced PA group and remained constant in the Usual PA group. In order to plot all cognitive test results on the same graph, we converted the raw scores to z-scores (Fig. 6). Changes in the standardized CVLT Total and Long Delay were not different between groups, $p=0.210$ and $p=0.169$, respectively. In contrast, the Enhanced PA group improved significantly on the D-KEFS CWI as compared to the Usual PA group, $p=0.022$. Improvement on the D-KEFS CWI was positively correlated with change in $\mathrm{VO}_{2}$ peak when both groups were pooled, $r=-0.449, p=0.032$ (Fig. 7) but was not significantly correlated with change in MVPA, $r=-0.221, p=0.312$.

\section{DISCUSSION}

The major finding of this study is that increased brain glucose metabolism and executive function following 26 weeks of aerobic exercise training was associated with CRF improvement. Furthermore, executive function was improved in participants randomized to Enhanced PA as compared to those maintaining Usual PA. The present results are consistent with mounting evidence that aerobic exercise training has positive effects on brain structure and function in late-middle aged adults with normal cognition [6, 41-46]. Importantly, we expand on this

Table 3

Baseline and post-intervention cognitive scores

\begin{tabular}{|c|c|c|c|c|c|c|}
\hline & \multicolumn{3}{|c|}{ Usual PA } & \multicolumn{3}{|c|}{ Enhanced PA } \\
\hline & Baseline & Post & Effect Size & Baseline & Post & Effect Size \\
\hline MMSE, a.u. & $29.67(0.49)$ & $29.08(1.24)$ & 0.626 & $29.55(0.69)$ & $29.55(0.52)$ & 0.000 \\
\hline CVLT Total, words & $49.58(11.06)$ & $53.33(9.18)$ & 0.369 & $53.18(7.99)$ & $60.64(9.04)$ & 0.874 \\
\hline CVLT Long Delay, words & $10.83(2.83)$ & $11.42(3.26)$ & 0.193 & $12.00(2.72)$ & $14.00(1.73)$ & 0.877 \\
\hline D-KEFS CWI, seconds & $60.00(11.71)$ & $60.00(11.99)$ & 0.000 & $64.82(15.11)$ & $57.64(11.96)$ & 0.527 \\
\hline
\end{tabular}

Cognitive test scores at baseline and post-intervention; within-group Cohen's $d$ effect sizes for Usual and Enhanced PA groups. Data are presented as mean (standard deviation). Abbreviations: physical activity (PA); Mini-Mental State Examination (MMSE); California Verbal Learning Test-II (CVLT); Delis-Kaplan Executive Function System Color Word Interference (D-KEFS CWI). 


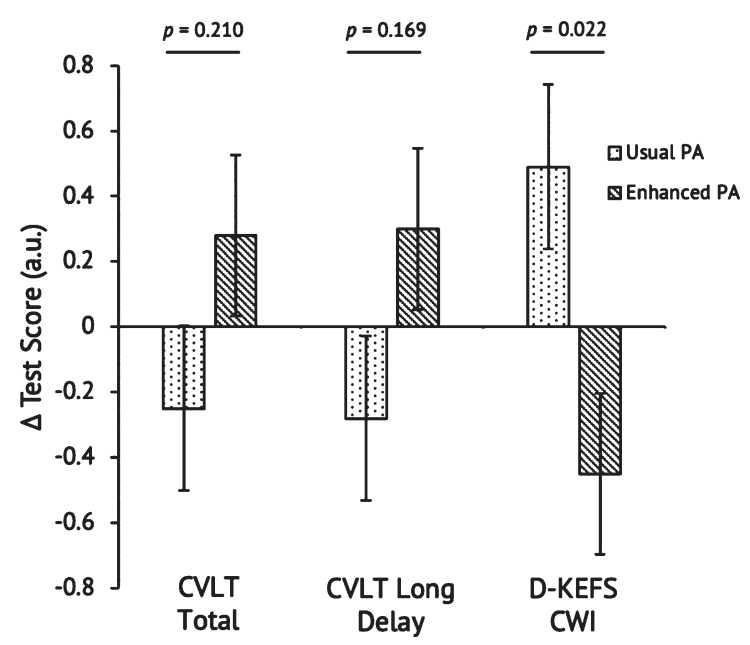

Fig. 6. Change in CVLT Total and Delayed scores, and D-KEFS Color Word Interference scores after the 26-week intervention. Raw scores were converted to $z$-scores so that the three tests could be plotted along the same y-axis. Independent samples $t$-tests were conducted to determine the statistical significance of the difference between Usual PA $(n=12)$ and Enhanced PA $(n=11)$. Higher scores indicate better performance for the CVLT whereas lower scores indicate better performance for the D-KEFS CWI. Abbreviations: California Verbal Learning Test-II Total Score (CVLT Total); California Verbal Learning Test-II Long Delay (CVLT Long Delay); Delis-Kaplan Executive Function System Color Word Interference (D-KEFS CWI); physical activity (PA).

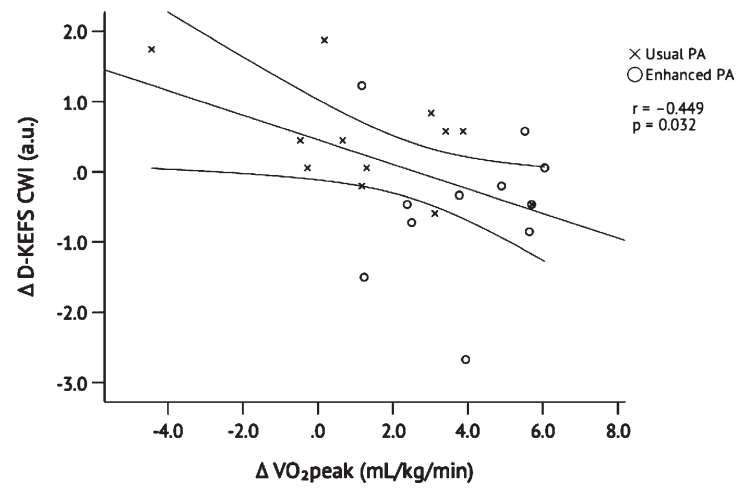

Fig. 7. Pearson's correlation between in changes in D-KEFS Color Word Interference scores and $\mathrm{VO}_{2}$ peak after 26 weeks of Usual PA $(n=12)$ or Enhanced PA $(n=11)$. The y-axis $\Delta$ D-KEFS values are $z$-scores calculated to align with the $z$-scores plotted in Figure 5. Abbreviations: Delis-Kaplan Executive Function System (DKEFS); peak oxygen consumption $\left(\mathrm{VO}_{2}\right.$ peak); physical activity (PA).

body of literature by demonstrating beneficial effects of aerobic exercise on brain glucose metabolism in a sample enriched for AD risk. Furthermore, we demonstrate that six months of aerobic exercise training is feasible and acceptable in this population with a session adherence rate of over $99 \%$. A particularly notable finding of this study is that the Enhanced PA group successfully increased CRF and MVPA and decreased sedentary behavior, which is likely attributable to the study design and excellent adherence on the part of the participants.

Our findings are in accordance with a previous study demonstrating that aerobic exercise training may benefit brain glucose metabolism. Porto and colleagues reported that 24 weeks of aerobic exercise training increased PCC glucose metabolism among individuals with MCI [47]. Their exercise training was similar in session duration, intensity, and intervention duration, but was lower in total training volume (i.e., two sessions/week) and the improvement in CRF was approximately half of that observed in our Enhanced PA group. It is possible that more frequent exercise sessions would have resulted in more robust improvement, but also possible that individuals who have already progressed to MCI have less capacity to improve CRF than our cognitively normal cohort [48]. Robinson et al. reported that 12 weeks of high-intensity interval exercise in cognitively normal, older adults resulted in no change in PCC glucose metabolism despite an increase in the left parietal and temporal lobes and right caudate nucleus [49]. Furthermore, that study did not reveal any correlation between CRF and brain glucose uptake. There may be several explanations for our divergent findings. A recent meta-analysis indicates exercise training for more than 52 total hours is needed to observe change in cognitive function [50]. Robinson et al.'s exercise intervention lasted only 12 weeks and consisted of approximately 35 total hours of training, which is substantially less than our 26-week intervention of approximately 58 hours of training. Furthermore, the type of training performed in their study included high intensity interval training, using 4 intervals of 4 minutes at $90 \%$ of $\mathrm{VO}_{2}$ peak with 3 minutes of low intensity recovery between intervals; it is possible that moderate intensity PA is sufficient for promoting brain glucose metabolism [29]. More research is needed to understand how to better customize exercise dose (i.e., frequency, intensity, and duration) for targeted improvement in brain glucose metabolism.

Previous findings from our laboratory [29] show that engagement in physical activity is positively associated with brain glucose metabolism in a cohort similar to the present one. The results of this pilot intervention extend those observational, cross-sectional findings by demonstrating that CRF contributes to the relationship between structured 
exercise training and brain glucose metabolism. The data we report here suggest that improvement in CRF contributes to enhanced brain glucose metabolism in the PCC, a region in which hypometabolism is a feature of the earliest stages of $\mathrm{AD}$ [51]. In that previous investigation by our group [29], engagement in higher amounts of moderate intensity PA was positively associated with PCC glucose metabolism in a stepwise fashion: individuals in the highest two tertiles of minutes of moderate PA (average of 68.1 and 105.7 minutes/day, respectively) had significantly greater brain glucose metabolism than those in the lowest tertile. As a result of the intervention, the Enhanced PA group in the present study increased MVPA to an amount consistent with detectably higher brain glucose metabolism (i.e., 90 minutes/day of MVPA) [29]. While there was no significant correlation between change in PCC metabolism and MVPA, there was with $\mathrm{VO}_{2}$ peak, suggesting that engagement in MVPA should aim to increase CRF in order to benefit PCC glucose metabolism. This is supported by previous research from our laboratory [52] and others [12] demonstrating CRF to be a stronger predictor than PA for a variety of outcomes that are linked with AD.

Our results demonstrate a significant effect of aerobic exercise training on a measure of executive function in our at-risk cohort. Enhancing executive function prior to AD-related cognitive decline may provide some measure of resilience to the disease. Our findings are in accordance with aerobic exercise interventions resulting in improved performance on tests of executive function in individuals with normal cognition [42, 45, 53, 54], although contradictions have been reported [55]. A recent study reported that cognition and executive function also improved among individuals with MCI after six weeks of aerobic exercise training [56]. On the other hand, two recent six-month aerobic exercise interventions did not result in significant change in executive function among individuals with $\mathrm{AD}[48,57]$. In the context of the existing $\mathrm{AD}$ literature, findings from the present study reinforce the idea that aerobic exercise may have a more substantial impact on executive function when implemented prior to disease onset or early in the disease course [58-60]. Further research should aim to determine the mechanisms underpinning the relationship between exercise training, $\mathrm{VO}_{2}$ peak, and executive function in individuals at risk for AD.

Our findings demonstrate no significant effect of exercise training on the CVLT. This is surprising given studies that report improvement in episodic memory after aerobic exercise training, even in individuals with MCI $[61,62]$. It is possible that the mechanisms underpinning the relationship between CRF and executive function do not impact episodic memory to the same degree, as we did not observe a significant correlation between performance on the CVLT and $\mathrm{VO}_{2}$ peak. Chapman et al. also found no improvement on the CVLT after 12 weeks of three sessions per week of moderate intensity aerobic exercise training among older individuals with normal cognition, nor did they observe a significant increase in CRF [55]. It is important to note that, while not statistically significant, our Enhanced PA group did increase their CVLT scores on average. It is likely that the present study was underpowered to detect such a change in episodic memory and that a ceiling effect may have been observed since mean scores were high.

There are limitations to the present study that must be considered. Due to budgetary constraints, the staff who conducted the neuropsychological evaluations was not blinded to the participants' treatment assignment. This is a potential source of bias that could have affected the cognitive outcomes of the study. However, this limitation could not have affected findings on brain glucose metabolism, especially as the technicians conducting the FDG-PET scans were blinded. Secondly, this was a pilot study primarily designed to establish feasibility of the protocol; further research including a larger sample is needed to validate the present findings and to allow determination of effects that may be specific to sex or genetic risk profile, as preliminary evidence shows that individuals at high genetic risk differ on brain glucose metabolism as a function of CRF [63]. Thirdly, the Usual PA group did not have weekly interactions with an exercise trainer, and thus may not have received the same degree of structured social engagement as the Enhanced PA group. Social engagement is known to impact cognitive outcomes [64], and such an effect was observed in an exercise training study [65]. Finally, practice effects could have affected the validity of the cognitive testing since tests were given just six months apart.

In conclusion, increased CRF following this sixmonth intervention was associated with enhanced brain glucose metabolism in the PCC, a region linked to $\mathrm{AD}$, and cognition among late-middle-aged individuals at risk for $\mathrm{AD}$. If these findings are supported by a larger-scale study, this would provide strong evidence that adults at risk for $\mathrm{AD}$ may enhance brain function and cognition by engaging in aerobic exercise training. 


\section{ACKNOWLEDGMENTS}

This work was supported by grants from the Alzheimer's Association (NIRGD-305257), the Extendicare Foundation, and the National Institute on Aging (K23 AG045957, R01 AG027161, P50 AG033514, and UL1RR025011). Ryan J. Dougherty was supported by a National Research Service Award from the National Institute on Aging of the National Institutes of Health under Award Number F31AG062009. We thank the staff and study participants of the Wisconsin Registry for Alzheimer's Prevention and the Wisconsin Alzheimer's Disease Research Center.

\section{CONFLICT OF INTEREST}

The authors have no conflict of interest to report.

\section{REFERENCES}

[1] Hebert LE, Weuve J, Scherr PA, Evans DA. Alzheimer disease in the United States (2010-2050) estimated using the 2010 census. Neurology. 2013;80(19):1778-83.

[2] Sperling RA, Jack CR, Aisen PS. Testing the Right Target and Right Drug at the Right Stage. Sci Transl Med. 2011;3(111):111 cm 33 .

[3] Adlard PA, Perreau VM, Pop V, Cotman CW. Voluntary Exercise Decreases Amyloid Load in a Transgenic Model of Alzheimer's Disease. J Neurosci. 2005;25(17):4217-21.

[4] Wu C-W, Chang Y-T, Yu L, Chen H, Jen CJ, Wu S-Y, et al. Exercise Enhances the Proliferation of Neural Stem Cells and Neurite Growth and Survival of Neuronal Progenitor Cells in Dentate Gyrus of Middle-Aged Mice. J Appl Physiol. 2008;105(5):1585-94.

[5] Zhang X, He Q, Huang T, Zhao N, Liang F, Xu B, et al. Treadmill Exercise Decreases $A \beta$ Deposition and Counteracts Cognitive Decline in APP/PS1 Mice, Possibly Via Hippocampal Microglia Modifications. Front Aging Neurosci. 2019;11(78):1-12.

[6] Erickson KI, Voss MW, Prakash RS, Basak C, Szabo A, Chaddock L, et al. Exercise Training Increases Size of Hippocampus and Improves Memory. Proc Natl Acad Sci. 2011;108:3017-22.

[7] Erickson KI, Prakash RS, Voss MW, Chaddock L, Hu L, Morris KS, et al. Aerobic Fitness is Associated With Hippocampal Volume in Elderly Humans. Hippocampus. 2009;19(10):1030-9.

[8] Baker LD, Frank LL, Foster-Schubert K, Green PS, Wilkinson CW, McTiernan A, et al. Effects of Aerobic Exercise on Mild Cognitive Impairment: A Controlled Trial. Arch Neurol. 2010;67(1):71-9.

[9] Colcombe S, Kramer AF. Fitness Effects on the Cognitive Function of Older Adults: A Meta-Analytic Study. Psychol Sci. 2003;14(2):125-30.

[10] Pereira AC, Huddleston DE, Brickman AM, Sosunov AA, Hen R, McKhann GM, et al. An in vivo Correlate of Exercise-Induced Neurogenesis in the Adult Dentate Gyrus. Proc Natl Acad Sci. 2007;104(13):5638-43.
[11] Maass A, Düzel S, Goerke M, Becke A, Sobieray U, Neumann K, et al. Vascular Hippocampal Plasticity After Aerobic Exercise in Older Adults. Mol Psychiatry. 2015;20(5):585-93.

[12] Voss MW, Weng TB, Burzynska AZ, Wong CN, Cooke GE, Clark R, et al. Fitness, but not Physical Activity, is Related to Functional Integrity of Brain Networks Associated with Aging. NeuroImage. 2016;131:113-25.

[13] Colcombe SJ, Erickson KI, Raz N, Webb AG, Cohen NJ, McAuley E, et al. Aerobic Fitness Reduces Brain Tissue Loss in Aging Humans. J Gerontol A Biol Sci Med Sci. 2003;58(2):176-80.

[14] Johnson NF, Kim C, Clasey JL, Bailey A, Gold BT. Cardiorespiratory Fitness is Positively Correlated with Cerebral White Matter Integrity in Healthy Seniors. NeuroImage. 2012;59(2):1514-23.

[15] Small GW, Ercoli LM, Silverman DHS, Huang S-C, Komo S, Bookheimer SY, et al. Cerebral Metabolic and Cognitive Decline in Persons at Genetic Risk for Alzheimer's Disease. Proc Natl Acad Sci. 2000;97(11):6037-42.

[16] Cupples LA, Farrer LA, Sadovnick AD, Relkin N, Whitehouse P, Green RC. Estimating Risk Curves for First-Degree Relatives of Patients with Alzheimer's Disease: The REVEAL Study. Genet Med. 2004;6:192-6.

[17] Mosconi L, Tsui WH, Herholz K, Pupi A, Drzezga A, Lucignani G, et al. Multicenter Standardized 18F-FDG PET Diagnosis of Mild Cognitive Impairment, Alzheimer's Disease, and Other Dementias. J Nucl Med. 2008;49(3): 390-8.

[18] Protas HD, Chen K, Langbaum JBS, Fleisher AS, Alexander GE, Lee W, et al. Posterior Cingulate Glucose Metabolism, Hippocampal Glucose Metabolism, and Hippocampal Volume in Cognitively Normal, Late-Middle-Aged Persons at 3 Levels of Genetic Risk for Alzheimer Disease. JAMA Neurol. 2013;70(3):320-5.

[19] Grober E, Hall CB, Lipton RB, Zonderman AB, Resnick SM, Kawas C. Memory Impairment, Executive Dysfunction, and Intellectual Decline in Preclinical Alzheimer's Disease. J Int Neuropsychol Soc. 2008;14(2):266-78.

[20] Harrington MG, Chiang J, Pogoda JM, Gomez M, Thomas $\mathrm{K}$, Marion SD, et al. Executive Function Changes Before Memory in Preclinical Alzheimer's Pathology: A Prospective, Cross-Sectional, Case Control Study. PLoS One. 2013;8(11):e79378.

[21] Johnson SC, Koscik RL, Jonaitis EM, Clark LR, Mueller $\mathrm{KD}$, Berman SE, et al. The Wisconsin Registry for Alzheimer's Prevention: A Review of Findings and Current Directions. Alzheimers Dement Diagn Assess Dis Monit. 2018;10:130-42.

[22] McKay GJ, Silvestri G, Chakravarthy U, Dasari S, Fritsche LG, Weber BH, et al. Variations in Apolipoprotein E Frequency With Age in a Pooled Analysis of a Large Group of Older People. Am J Epidemiol. 2011;173(12):135764.

[23] Mayer CJ, Steinman L, Williams B, LoGerfo J, Topolski TD. Developing a Telephone Assessment of Physical Activity (TAPA) Questionnaire for Older Adults. Prev Chronic Dis. 2007;5(1):1-7.

[24] Nelson ME, Rejeski WJ, Blair SN, Duncan PW, Judge JO, King AC, et al. Physical Activity and Public Health in Older Adults: Recommendation from the American College of Sports Medicine and the American Heart Association. Med Sci Sports Exerc. 2007;39(8):1435-45.

[25] Borg G. Psychophysical Bases of Perceived Exertion. Med Sci Sports Exerc. 1982;14(5):377-81. 
[26] ACSM's Guidelines for Exercise Testing and Prescription (Tenth Edition). 10th ed. Wolters Kluwer; 2018.

[27] Troiano RP, Berrigan D, Dodd KW, Mâsse LC, Tilert T, Mcdowell M. Physical Activity in the United States Measured by Accelerometer. Med Sci Sports Exerc. 2008;40(1):181-8.

[28] Lyden K, Keadle SK, Staudenmayer J, Freedson PS. A Method to Estimate Free-Living Active and Sedentary Behavior from an Accelerometer. Med Sci Sports Exerc. 2014;46(2):386-97.

[29] Dougherty RJ, Schultz SA, Kirby TK, Boots EA, Oh JM, Edwards D, et al. Moderate Physical Activity Is Associated with Cerebral Glucose Metabolism in Adults at Risk for Alzheimer's Disease. J Alzheimers Dis. 2017;58(4): 1089-97.

[30] Staudenmayer J, Pober D, Crouter S, Bassett D, Freedson P. An Artificial Neural Network to Estimate Physical Activity Energy Expenditure and Identify Physical Activity Type from an Accelerometer. J Appl Physiol. 2009;107(4): 1300-7.

[31] Okonkwo OC, Schultz SA, Oh JM, Larson J, Cook D, Koscik R, et al. Physical Activity Attenuates Age-Related Biomarker Alterations in Preclinical AD. Neurology. 2014;83:1753-60

[32] Okonkwo OC, Oh JM, Koscik R, Jonaitis E, Cleary CA, Dowling NM, et al. Amyloid Burden, Neuronal Function, and Cognitive Decline in Middle-Aged Adults at Risk for Alzheimer's Disease. J Int Neuropsychol Soc JINS. 2014;20(4):422-33.

[33] Johnson SC, Christian BT, Okonkwo OC, Oh JM, Harding $\mathrm{S}, \mathrm{Xu} \mathrm{G}$, et al. Amyloid Burden and Neural Function in People at Risk for Alzheimer's Disease. Neurobiol Aging. 2014;35(3):576-84.

[34] Jagust WJ, Bandy D, Chen K, Foster NL, Landau SM, Mathis CA, et al. The Alzheimer's Disease Neuroimaging Initiative Positron Emission Tomography Core. Alzheimers Dement. 2010;6(3):221-9.

[35] Floberg JM, Mistretta CA, Weichert JP, Hall LT, Holden JE, Christian BT. Improved Kinetic Analysis of Dynamic PET Data with Optimized HYPR-LR: Improved Dynamic PET Analysis with HYPR-LR. Med Phys. 2012;39(6): 3319-31.

[36] Sperling RA, Aisen PS, Beckett LA, Bennett DA, Craft S, Fagan AM, et al. Toward Defining the Preclinical Stages of Alzheimer's Disease: Recommendations from the National Institute on Aging-Alzheimer's Association Workgroups on Diagnostic Guidelines for Alzheimer's Disease. Alzheimers Dement. 2011;7(3):280-92.

[37] Landau SM, Harvey D, Madison CM, Koeppe RA, Reiman EM, Foster NL, et al. Associations Between Cognitive, Functional, and FDG-PET Measures of Decline in AD and MCI. Neurobiol Aging. 2011;32(7):1207-18.

[38] Folstein MF, Folstein SE, McHugh PR. "Mini-Mental State": A Practical Method for Grading the Cognitive State of Patients for the Clinician. J Psychiatr Res. 1975;12(3): 189-98.

[39] Delis DC, Kramer JH, Kaplan E, Ober BA. California Verbal Learning Test. San Antonio: Psychological Corporation; 2000.

[40] Delis DC, Kaplan E, Kramer JH. Delis-Kaplan Executive Function System. In San Antonio, TX: Psychological Corporation; 2001.

[41] Voss MW, Heo S, Prakash RS, Erickson KI, Alves H, Chaddock L, et al. The Influence of Aerobic Fitness on Cerebral
White Matter Integrity and Cognitive Function in Older Adults: Results of a One-Year Exercise Intervention. Hum Brain Mapp. 2013;34(11):2972-85.

[42] Smiley-Oyen AL, Lowry KA, Francois SJ, Kohut ML, Ekkekakis P. Exercise, Fitness, and Neurocognitive Function in Older Adults: The "Selective Improvement" and "Cardiovascular Fitness" Hypotheses. Ann Behav Med. 2008;36(3):280-91.

[43] Dustman RE, Ruhling RO, Russell EM, Shearer DE, Bonekat HW, Shigeoka JW, et al. Aerobic Exercise Training and Improved Neuropsychological Function of Older Individuals. Neurobiol Aging. 1984;5(1):35-42.

[44] Hawkins HL, Kramer AF, Capaldi D. Aging, Exercise, and Attention. Psychol Aging. 1992;7(4):643-53.

[45] Kramer AF, Hahn S, Cohen NJ, Banich MT, McAuley E, Harrison CR, et al. Ageing, Fitness and Neurocognitive Function. Nature. 1999;400(6743):418-9.

[46] Albinet CT, Boucard G, Bouquet CA, Audiffren M. Increased Heart Rate Variability and Executive Performance After Aerobic Training in the Elderly. Eur J Appl Physiol. 2010;109(4):617-24.

[47] Henrique de Gobbi Porto F, Martins Novaes Coutinho A, Lucia de Sá Pinto A, Gualano B, Luís de Souza Duran F, Prando S, et al. Effects of Aerobic Training on Cognition and Brain Glucose Metabolism in Subjects with Mild Cognitive Impairment. J Alzheimers Dis. 2015;46(3):747-60.

[48] Morris JK, Vidoni ED, Johnson DK, Van Sciver A, Mahnken JD, Honea RA, et al. Aerobic Exercise for Alzheimer's Disease: A Randomized Controlled Pilot Trial. PLoS ONE. 2017;12(2):1-14.

[49] Robinson MM, Lowe VJ, Nair KS. Increased Brain Glucose Uptake After 12 Weeks of Aerobic High-Intensity Interval Training in Young and Older Adults. J Clin Endocrinol Metab. 2017;103(1):221-7.

[50] Gomes-Osman J, Cabral DF, Morris TP, McInerney K, Cahalin LP, Rundek T, et al. Exercise for Cognitive Brain Health in Aging: A Systematic Review for an Evaluation of Dose. Neurol Clin Pract. 2018;8(3):257-65.

[51] Minoshima S, Giordani B, Berent S, Frey KA, Foster NL, Kuhl DE. Metabolic Reduction in the Posterior Cingulate Cortex in Very Early Alzheimer's Disease. Ann Neurol. 1997;42(1):85-94.

[52] Dougherty RJ, Boots EA, Lindheimer JB, Stegner AJ, Van Riper S, Edwards DF, et al. Fitness, Independent of Physical Activity Is Associated with Cerebral Blood Flow in Adults at Risk for Alzheimer's Disease. Brain Imaging Behav. 2019; 10.1007/s11682-019-00068-w

[53] Jonasson LS, Nyberg L, Kramer AF, Lundquist A, Riklund K, Boraxbekk C-J. Aerobic Exercise Intervention, Cognitive Performance, and Brain Structure: Results from the Physical Influences on Brain in Aging (PHIBRA) Study. Front Aging Neurosci. 2017;8(336):1-15.

[54] Masley S, Roetzheim R, Gualtieri T. Aerobic Exercise Enhances Cognitive Flexibility. J Clin Psychol Med Settings. 2009;16(2):186-93.

[55] Chapman SB, Aslan S, Spence JS, DeFina LF, Keebler MW, Didehbani N, et al. Shorter Term Aerobic Exercise Improves Brain, Cognition, and Cardiovascular Fitness in Aging. Front Aging Neurosci. 2013;5(75):1-9.

[56] Amjad I, Toor H, Niazi IK, Afzal H, Jochumsen M, Shafique M, et al. Therapeutic Effects of Aerobic Exercise on EEG Parameters and Higher Cognitive Functions in Mild Cognitive Impairment Patients. Int $\mathbf{J}$ Neurosci. 2019;129(6):551-62. 
[57] Yu F, Vock DM, Barclay TR. Executive Function: Responses to Aerobic Exercise in Alzheimer's Disease. Geriatr Nurs N Y N. 2018;39(2):219-24.

[58] Voss MW, Erickson KI, Prakash RS, Chaddock L, Kim JS, Alves H, et al. Neurobiological Markers of Exercise-Related Brain Plasticity in Older Adults. Brain Behav Immun. 2013;28:90-9.

[59] Voss MW, Prakash RS, Erickson KI, Basak C, Chaddock L, Kim JS, et al. Plasticity of Brain Networks in a Randomized Intervention Trial of Exercise Training in Older Adults. Front Aging Neurosci. 2010;2(32):1-17.

[60] Cespón J, Miniussi C, Pellicciari MC. Interventional Programmes to Improve Cognition During Healthy and Pathological Ageing: Cortical Modulations and Evidence for Brain Plasticity. Ageing Res Rev. 2018;43:81-98.

[61] Smith JC, Nielson KA, Antuono P, Lyons J-A, Hanson RJ, Butts AM, et al. Semantic Memory Functional MRI and Cognitive Function After Exercise Intervention in Mild Cognitive Impairment. J Alzheimers Dis. 2013;37(1): 197-215.
[62] Nagamatsu LS, Chan A, Davis JC, Beattie BL, Graf P, Voss MW, et al. Physical Activity Improves Verbal and Spatial Memory in Older Adults with Probable Mild Cognitive Impairment: A 6-Month Randomized Controlled Trial. J Aging Res. 2013;2013:1-10.

[63] Deeny SP, Winchester J, Nichol K, Roth SM, Wu JC, Dick M, et al. Cardiovascular Fitness Is Associated with Altered Cortical Glucose Metabolism During Working Memory in $\varepsilon 4$ Carriers. Alzheimers Dement. 2012;8(4):352-6.

[64] Fratiglioni L, Paillard-Borg S, Winblad B. An Active and Socially Integrated Lifestyle in Late Life Might Protect Against Dementia. Lancet Neurol. 2004;3(6):343-53.

[65] Mortimer JA, Ding D, Borenstein AR, DeCarli C, Guo $\mathrm{Q}, \mathrm{Wu} \mathrm{Y}$, et al. Changes in Brain Volume and Cognition in a Randomized Trial of Exercise and Social Interaction in a Community-Based Sample of Non-Demented Chinese Elders. J Alzheimers Dis. 2012;30(4):757-66. 